\title{
Formación docente y narrativas pedagógicas: una apuesta de trabajo en red desde la extensión universitaria
}

Daniel Hugo Suárez

dhsuarez@filo.uba.ar

Paula Valeria Dávila

paulavdavila@gmail.com

Agustina Argnani

agustina.argnani@gmail.com

\section{Yanina Caressa}

ycaressa@gmail.com

Universidad de Buenos Aires, Argentina.
A 100 años de la Reforma Universitaria de 1918

Intervenciones

RECEPCIÓN: 29/06/17

ACEPTACIÓN FINAL: 24/10/17

\section{Resumen}

El Programa de Extensión Universitaria de la Facultad de Filosofía y Letras de la Universidad de Buenos Aires (FFYL-UBA) "Red de Formación Docente y Narrativas Pedagógicas" se teje en torno de relatos de experiencias producidos a través de un dispositivo de investigación-formación-acción docente en red: la documentación narrativa de experiencias pedagógicas. Dicho dispositivo supone la indagación de las propias experiencias pedagógicas en procesos de formación horizontal entre colegas por medio de la escritura, la lectura, la conversación, la publicación y la circulación de sus relatos de experiencia. Esto constituye una estrategia de formación en la medida en que permite resignificar y reorientar las prácticas de manera sistemática, reflexiva y crítica. Pero al mismo tiempo, este proceso supone la reconstrucción, disposición pública y deliberación colectiva en torno del saber pedagógico puesto en juego en las prácticas de docentes y de otros actores educativos.

Palabras clave

- Formación docente

- Documentación narrativa

- Trabajo en red

- Investigación (auto)biográfica y narrativa

\section{Resumo}

O Programa de Extensão Universitária (FFYL-UBA) "Rede de Formação Docente e Narrativas Pedagógicas" se constrói em torno de relatos de experiências produzidos por meio de um dispositivo de pesquisa-formação-ação docente em rede: a documentação narrativa de experiências pedagógicas. Tal dispositivo envolve a indagação de suas próprias experiências pedagógicas em processos de formação horizontal entre colegas, por meio da escritura, leitura, conversação, publicação e da circulação de seus relatos da experiência. Isto constitui uma estratégia de formação na medida em que permite ressignificar e reorientar as práticas de forma sistemática, reflexiva e crítica. Mas, ao mesmo tempo, este processo envolve a reconstrução, disposição publica e deliberação coletiva em torno do saber pedagógico colocado em jogo nas práticas dos docentes e dos outros atores educativos.

Palavras-chave

- Formação docente

- Documentação narrativa

- Trabalho em rede

- Pesquisa (auto)biográfica e narrativa
Para citación de este artículo

Suárez, D. H; Dávila, P. V.; Argnani, A. y Caressa, Y. (2017). Formación docente y narrativas pedagógicas: una apuesta de trabajo en red desde la extensión universitaria. Revista + E versión en línea, 7(7), 244-253. Santa Fe, Argentina: Ediciones UNL. 


\section{Intervención, formación e investigación pedagógica} desde la extensión universitaria

En este artículo nos interesa ofrecer algunas coordenadas, perspectivas y pistas para pensar la extensión universitaria como parte constitutiva, como dimensión integral del trabajo académico de docencia e investigación que venimos desarrollando algunos de los que habitamos y transitamos esta institución. Este escrito incluye la revisión de la idea de "función" como una noción que alude a lo fijo y predefinido de antemano y que, como consecuencia, separa en componentes aislados las tareas de enseñanza, investigación y extensión. Desde el Programa de Extensión Universitaria "Red de Formación Docente y Narrativas Pedagógicas" de la UBA ${ }^{1}$ se propone una reformulación de estas nociones: a partir del desarrollo de dispositivos de conversación y de coproducción de saber pedagógico en los que el trabajo desde la Universidad no se hace sobre los sujetos (en este caso, educadores de distintos ámbitos) sino junto con ellos.

El Programa enlaza una red de colaboración y trabajo coparticipado a colectivos de docentes narradores ${ }^{2}$ situados en diferentes espacios institucionales, organizacionales y sociales (que denominamos "nodos"). ${ }^{3}$ Esta "red de redes" permite generar espacios de confluencia y articulación, promoviendo así la transmisión e intercambio de saberes pedagógicos con vistas a fortalecer y potenciar los procesos formativos de los actores individuales y colectivos involucrados en cada uno de sus nodos. La Red se orienta a activar y recrear la memoria pedagógica de las prácticas escolares mediante la producción, circulación y publicación de relatos de experiencia pedagógica escritos por docentes, estudiantes y educadores. Esos documentos narrativos elaborados desde la investigación del propio mundo escolar permiten que el saber y el discurso pedagógicos construidos por los docentes se inscriban e intervengan en el debate público y especializado sobre la educación y la escuela. Al mismo tiempo que investiga, la Red de docentes narradores despliega trayectos de formación entre pares y experiencias colectivas de desarrollo profesional docente centrado en la indagación narrativa y (auto) biográfica de la práctica y el trabajo docente.

Desde una perspectiva particular de investigación-formaciónacción, ${ }^{4}$ la Red se sostiene como una iniciativa de intervención político-pedagógica de formación e investigación orientada a involucrar de manera activa a los docentes en la producción y reconstrucción crítica de sus saberes pedagógicos. Y posibilita, a su vez, la disposición pública de un corpus de relatos pedagógicos que interpelan y tensionan los discursos y la teoría educativa pública. Para ello, desarrollamos e implementamos un dispositivo de trabajo que genera relaciones horizontales entre docentes e investigadores y se orienta a la producción de relatos de experiencias escritos por los propios docentes: la documentación narrativa de experiencias pedagógicas (Suárez, 2009). Dicho dispositivo propone la indagación de las propias experiencias pedagógicas, en procesos de formación horizontal entre colegas, por medio de la escritura, la lectura, la conversación, la publicación y la circulación de sus relatos de experiencia. Esto constituye una estrategia de formación en la medida en que permite resignificar y reorientar las prácticas de manera sistemática, reflexiva y crítica. Pero a la vez este proceso supone la reconstrucción, disposición pública y deliberación colectiva en torno del saber pedagógico puesto en juego en las prácticas de docentes y de otros actores educativos. Una vez que estos relatos circulan y son conocidos en comunidades de docentes lectores-escritores de narraciones pedagógicas y se vuelven públicamente disponibles, esas historias de enseñanza se constituyen en documentos pedagógicos.
1) Programa aprobado por el Consejo Directivo de la Facultad de Filosofía y Letras, UBA (Resolución № 1425 del año 2010).

2) Denominamos de este modo al conjunto de docentes que participa de instancias de escritura, lectura, intercambios y reflexión pedagógica en el marco del dispositivo de DNEP. La cantidad de integrantes, las formas de coordinación y las modalidades de encuentro y trabajo varían en función de las definiciones de cada colectivo y del Nodo al que pertenecen, en caso de formar parte de la Red en cuestión. Consideramos que dichos colectivos pueden ser pensados como formas organizativas de investigación, forma- ción e intervención en el campo de la pedagogía pública.

3) Los Nodos que actualmente componen la Red son: Instituto Superior de Formación Docente $N^{\circ} 100$ Unidad Académica ENSPA, Avellaneda; Instituto Superior de Formación Docente $N^{\circ} 54$ de Florencio Varela; Instituto Superior de Formación Docente $\mathrm{N}^{\circ}$ 11 de Lanús; Centro Universitario Municipal San Fernando; Colectivo de Docentes Narradores de la Facultad de Periodismo de la UNLP; Colectivo de directivos de Centros de Formación Profesional; Programa de Desarrollo Profesional Docente en Formación Profesional (ODET-UOCRA y FFyL UBA); Colectivo de Graduados en
Técnicos en Recreación del Instituto Superior de tiempo Libre y Recreación de la Ciudad Autónoma de Buenos Aires; Colectivo de docentes narradores de La Matanza; Colectivos de docentes narradores de Quilmes; Dirección General de Educación, Municipalidad de Avellaneda; Región 4. Berazategui. Modalidad de Psicología Comunitaria y Pedagogía Social (DGCyE de la Provincia de Buenos Aires), Región 4. Berazategui.

4) Como antecedentes que conjugan la producción participativa de conocimientos críticos con procesos de desarrollo profesional podemos identificar las propuestas de investigación educativa y pedagógica que se apoyan en los principios teóricos y metodológicos de la etnografía de la educación (Geertz; Batallán), de la investigación cualitativa y narrativa de las prácticas escolares (Bolívar; Connelly y Clandinin ; McEwan; Gudmundsdottir), de la investigación (auto)biográfica (Delory-Momberger; De Souza, Josso; Passeggi y De Souza; Pineau y Le Grand), de la investigación acción docente y de la investigación participante (Carr y Kemmis; Kincheloe; Anderson y Herr); estrategias de sistematización de experiencias (Jara) y de formación horizontal entre docentes (Suárez). 
En este proceso formativo y de investigación por la acción, los educadores se tornan autores de materiales documentales que dan cuenta de experiencias y saberes pedagógicos que los tienen como protagonistas y los interpelan como habitantes del mundo educativo. Los colectivos de narradores discuten los términos y promueven la recreación del pensamiento pedagógico. De este modo, generan procesos de toma de la palabra y de deliberación pública que significan el desarrollo profesional entre docentes a través del intercambio y la conversación acerca de lo reconstruido, narrado y documentado, y producen comprensiones e interpretaciones críticas sobre situaciones y procesos educativos que amplían, profundizan y ponen en tensión la reconstrucción de la memoria docente y el patrimonio pedagógico (Suárez, 2011 y 2014). Como señalábamos, la Red de Formación Docente y Narrativas Pedagógicas deviene de un conjunto de hilos, de hebras y de nudos que se van entramando, que forman un "tejido" de relatos de experiencias, y estos relatos son originados a través de un dispositivo específico que recorre un itinerario: la documentación narrativa de experiencias pedagógicas como modalidad particular de investigación-formación-acción coparticipada. Ahora bien, ¿por qué encarar el reto de instalarla como un programa de extensión universitaria? La propuesta era crear un espacio protegido de las dificultades que muchas veces condicionan el desarrollo y permanencia de redes por falta de reconocimiento institucional o de recursos y, a su vez, conformar un espacio al interior de la FFyL de la UBA que permitiera el encuentro, la escucha y el trabajo en colaboración entre docentes, investigadores, becarios, estudiantes y actores comunitarios preocupados por la indagación de las prácticas educativas y la intervención en el debate pedagógico desde la posición y la experiencia de los docentes. Otro motivo tenía que ver con que el ámbito de la extensión universitaria proporciona la posibilidad de sostener lazos y articulaciones entre los investigadores académicos y otros sujetos de conocimiento para la producción y validación del saber pedagógico; de trabajar con otros actores para la construcción de un conocimiento más sensible, sofisticado y potente; de generar relaciones de colaboración y de espacios coparticipativos (como otro modo de debatir y discutir acerca de la especificidad del trabajo docente) y de promover otras posiciones que disputen la configuración de las relaciones y jerarquías políticoinstitucionales establecidas en el campo educativo.

En América Latina se vienen acrecentando las experiencias de este tipo, y se advierte en la actualidad un significativo impulso del desarrollo de movimientos de docentes decididos a intervenir en el campo pedagógico a fin de ensayar nuevas formas para transformación democrática en educación a través de la reconstrucción del saber pedagógico y la construcción de conocimiento. En Argentina, por su parte, se están desarrollando desde el año 2000 diversas formas de organización y de trabajo pedagógico entre educadores orientadas a la investigación, la formación y la elaboración colectiva, descentrada y plural de nuevos discursos para la educación y la pedagogía. Redes y colectivos docentes diseñan y llevan a la práctica líneas de acción diversas y articuladas y estrechan vínculos de trabajo colaborativo con otras instituciones y organizaciones (Suárez y Argnani, 2011). Sin embargo, las iniciativas en que la Universidad compromete su participación decisiva desde una lógica de coformación con otros sujetos y actores del campo pedagógico han sido escasas. A partir de nuestro propio proceso de experimentación metodológica y de una serie de estudios llevados a cabo en sucesivos proyectos de investigación UBACyT, ${ }^{5}$ hemos identificado múltiples dificultades y obstáculos relacionados con la habilitación de espacios y tiempos para el trabajo colaborativo entre diversos actores del campo educativo y la Universidad. Muchos docentes y educadores consideran a la mayor parte de la investigación educativa desvinculada de las preguntas y problemas surgidos en las prácticas escolares. En este sentido, resulta limitada la articulación entre la investigación educativa desarrollada desde ámbitos académicos y el mundo de las prácticas pedagógicas y entre sus habitantes, es decir, investigadores y docentes. Maestros y profesores no ven en la investigación científica ni una lente para mirar, interpretar y recrear sus prácticas profesionales, ni un espacio de conversación e interpelación, ni la solución a los múltiples problemas prácticos con los que se enfrentan día a día. Frente a este tradicional "divorcio" entre investigación académica y experiencias y prácticas educativas, consideramos que resulta necesario fortalecer y desarrollar aquellos espacios destinados a poner en marcha procesos colaborativos y de articulación entre
5) Nos referimos a los siguientes proyectos, que hacen parte de la programación científica de la UBA: Proyecto de Investigación "La conformación del campo pedagógico: sentidos y disputas en torno de las desigualdades y diferencias en educación" (UBACyT 2014-2017 Grupos Consolidados).
Director: Dr. Daniel H. Suárez; Proyecto de Investigación "La construcción social del cambio educativo. Desigualdades y diferencias en el campo pedagógico" (UBACyT 2011-2014 F194). Directores: Dr. Daniel H. Suárez y Lic. Dora González; Proyecto de Investigación "La construcción social del cambio educativo. Una mirada político pedagógica a las continuidades y rupturas en las formas de lo escolar" (UBACyT 2008-2010 F028). Directores: Dr Daniel H. Suárez y Lic. Dora González; Proyecto de Investigación

"Pedagogos, docentes y relatos de experiencia. Saberes pedagógicos y experiencias formativas producidas en la trayectoria profesional" (UBACyT 2008-2010 F096). Directores: Dra. Andrea Alliaud y Dr. Daniel H. Suárez, todos ellos desarrollados en Instituto de investigaciones en Ciencias de la Educación (FFyL-UBA). 
docentes, educadores, institutos de formación docente, ámbitos municipales, comunitarios y la Universidad.

Como veremos más adelante, en un proyecto desarrollado en La Matanza, provincia de Buenos Aires, hemos tenido la oportunidad de repensar, tensionar y conmover la distancia entre la investigación académica y las prácticas educativas. Allí, docentes, directivos y supervisores/as junto a un grupo de investigadores/as de la Red se lanzaron a la tarea de co-construir conocimiento acerca de lo que acontece en las aulas del distrito con el impulso, el apoyo y el sostenimiento del gobierno local. En efecto, este proceso ha significado la confluencia, encuentro y disposición de diversas condiciones políticas, teóricas y metodológicas para su desarrollo como proyecto coparticipado. Esto ha implicado pensar las instancias de producción de conocimiento de manera colaborativa al involucrar activamente a los docentes en la reconstrucción de la propia palabra, de la memoria pedagógica vivida de la escuela y de las experiencias docentes y escolares que resultaron pedagógicamente significativas (Suárez, 2014). De este modo, se pone en tensión y se busca reformular las políticas de conocimiento y de reconocimiento pedagógicos articulando investigación educativa, práctica escolar, políticas educativas y formación docente. ${ }^{6}$

Pero, además, el desarrollo de la propuesta de la Red en el marco de la extensión universitaria ha permitido problematizar y revisar aspectos teórico-metodológicos de los dispositivos de investigación y formación que el equipo venía implementando para captar con mayor sutileza las tensiones, dificultades y demandas de los sujetos e instituciones participantes, así como las recreaciones y adaptaciones que los mismos realizaban al dispositivo de documentación narrativa al momento de ponerlo en marcha para el trabajo con sus colectivos docentes.

Asimismo, los debates y desarrollos teórico metodológicos desplegados fueron insumos importantes para reformulaciones sucesivas del programa de la materia de grado "Educación II. Análisis sistemático de los sistemas de educación formal y no formal. Problemas Pedagógicos Contemporáneos" de la carrera de Ciencias de la Educación (FFyL-UBA) y han sido incorporados como parte del encuadre teórico-metodológico en seminarios, créditos de investigación y créditos de práctica profesional para la Licenciatura en Ciencias de la Educación. Por su parte, las instancias de formación de posgrado (en maestrías y doctorados) hicieron posible para integrantes del equipo llevar adelante tesis ${ }^{7}$ centradas en el trabajo en el Programa de Extensión y, de esa manera, enriquecer las perspectivas teóricas, epistemológicas y metodológicas que contribuyeron a generar conocimiento productivo para repensar el trabajo de extensión. Al mismo tiempo, el trabajo de extensión contribuyó a cuestionar e interpelar el trabajo académico de investigación y el de formación en el posgrado. De este modo, la línea de investigación, el trabajo en la Red y las prácticas docentes que venimos desplegando se encuentran
6) Solo para tomar otro ejemplo de tal articulación, en el Nodo Sur de la Red, las propuestas y experiencias de documentación narrativa con diversos colectivos de esa zona (Ex Libris, Mirar la Escuela con Perspectiva de Género, Grupo en la Escuela 78, Docentes Jubilados, Colectivo de Equipos de Orientación Escolar) han sido desplegadas en articulación con la Subsecretaría de Educación, Políticas del Cuidado y de Género del Municipio de Quilmes (2011-2015), y el impacto en el desarrollo de políticas de esa subsecretaría ha sido de un importante alcance territorial.
7) Se trata de las tesis de Maestría en Educación, Pedagogías Críticas y Problemáticas Socioeducativas (FFyL/ UBA) de Agustina Argnani: "Redes pedagógicas, relatos de experiencias y formación docente. La Red de Formación Docente y Narrativas Pedagógicas, un estudio en caso"; de
Paula Dávila: "Escribir e interpretar la experiencia docente: la documentación narrativa de prácticas pedagógicas" y de Yanina Caressa: "Inspectores, investigación-formación-acción docente, red escolar y cambio educativo. Un estudio en caso en la Provincia de Buenos Aires". 
estrechamente vinculados y son insumos permanentes que permiten enriquecer, tensionar y revisar mutuamente los desarrollos del equipo como un conglomerado en el que no es factible operar distinciones tajantes entre las tres tareas fundamentales a las que son llamadas las universidades. Tradicionalmente, las prácticas de extensión han cristalizado modos específicos en que la universidad pública planteó su vinculación con diversos sujetos sociales. Concebida como una de las "funciones" de esta institución, los significados asociados a ella se articularon en torno del establecimiento de relaciones de colonialidad con los otros. Desde un lugar de presunta superioridad cultural, que suponía una preeminencia ética y prioridad política, la universidad "transfería", "extendía", difundía en la comunidad lega que la rodeaba -y que no accedía a sus propuestas de enseñanza o investigación- parte de los conocimientos científicos y tecnológicos que producía. Por el contrario, entendemos que el Programa plantea una perspectiva integral de las prácticas de extensión, investigación y docencia en vistas a desarrollar nuevas formas de enseñar, aprender e investigar en contacto más directo con los actores educativos, sus problemas, demandas y necesidades (Suárez, Dávila y Argnani, 2015).

\section{La formación docente desde la extensión: redes, territorios y escenarios dinámicos en permanente reconfiguración} Las redes pedagógicas que vienen conformándose en nuestra región son espacios de construcción de saberes y de sujetos en tanto configuran un entramado de experiencias, relaciones y sentidos en el cual los elementos heterogéneos, sujetos, instituciones y grupos redefinen su propia identidad desde la participación activa y la articulación con otros. La heterogeneidad, como elemento constitutivo de las redes, se enlaza en una trama de voces plurales, en una malla de nudos y nodos, de identidades en construcción, de procesos colectivos en desarrollo, de prácticas y conocimientos en constante revisión, de formas organizativas flexibles, que teje una comunidad pedagógica entre los sujetos e hilos que la conforman (Universidad Pedagógica Nacional, 2002; Martínez Boom y Peña Rodríguez, 2009).

A partir de la puesta en marcha del programa en el año 2010 hemos visualizado una mayor apropiación del dispositivo de trabajo por parte de las instituciones y organizaciones participantes, favorecido por un espacio de formación horizontal a través de procesos sistemáticos de indagación-formación-acción sobre las prácticas pedagógicas. En este sentido, el interés por conformar nuevos colectivos de docentes escritores/lectores y la efectiva incorporación de nuevos nodos y colectivos a la Red dan cuenta de ello. En tanto que favorece el intercambio de experiencias y el trabajo conjunto entre colectivos de docentes narradores, redes educativas, investigadores, docentes y estudiantes universitarios, la Red es percibida como un espacio de coinvestigación y formación que potencia el desarrollo de las actividades propias de cada institución u organización (Dávila y Argnani, 2015 y 2017). El trabajo en red dio lugar a que los docentes narradores involucrados participaran de diversas modalidades de encuentro, fueran presenciales o virtuales (de lectura entre pares, reuniones de coordinación, publicaciones colectivas, intercambio de experiencias o desarrollo de actividades conjuntas), que impulsaron la circulación de los relatos y el intercambio de experiencias vinculadas a la producción colectiva del saber pedagógico con integrantes de otras redes. Se esperaba que, al favorecer estructuras más flexibles y vínculos de colaboración, la Red permita a cada uno de los nodos extender y diversificar actividades, experimentar nuevos desarrollos y generar a la par nuevos espacios, tiempos y producciones. Acciones que difícilmente hubieran sido posibles de ser desarrolladas por cada institución de manera individual o por fuera del espacio de la Red, fuertemente apoyada en la generación de lazos y articulaciones. Es altamente valorada la posibilidad que ofrece el ámbito de la Red de participar e involucrarse en las líneas de acción y de investigación que llevan adelante otras redes que se encuentran en funcionamiento a nivel nacional y regional, como es la participación en encuentros, jornadas y actividades promovidas por otros colectivos y redes. ${ }^{8}$ Desde esta propuesta, la investigación-acción docente se plantea no simplemente como insumo para la investigación educativa sino, enmarcada en la extensión universitaria, como un espacio de producción colaborativa que pretende abrir una conversación entre los actores participantes y como un espacio de coformación y ecoformación ${ }^{9}$ e intervención en el debate pedagógico público. Ahora bien, en tanto consiste en una modalidad de producción colectiva de saberes, se requiere de criterios de validación distintos a los de las ciencias sociales tradicionales. Tales
8) Por ejemplo, la red participa del Colectivo Argentino de Docentes que hacen Investigación desde la Escuela (integrado por más de diez redes nacionales), que a su vez participa de la Red Iberoamericana de Colectivos y Redes de Maestros y Maestras que hacen Investigación e Innovación desde la Escuela y la Comunidad, en la que se congregan redes de España, Argentina, Colombia, Venezuela, México, Perú y Brasil.

9) Partiendo del abordaje biográfico y experiencial de la formación, el autor francés Gaston Pineau aborda los conceptos de autoformación, ecoformación y heteroformación como tres movimientos propios de la formación: el primero (auto-) remite al papel del sujeto en el proceso de formación, el cual es continuo y constante en la historia de la vida de cada individuo; (hetero-) refiere a la acción de los otros y la sociedad y (eco-) al medio ambiente y sus dimensiones físicas y naturales (De Souza, 2011). En nuestra propuesta retomamos la idea de ecoformación en vinculación a la de coformación (entendida esta como la formación junto con otros pares en relaciones de colaboración) para abordar los procesos formativos en tanto situados, anclados en territorios y espacios institucionales/organizacionales específicos. 


\section{6}

esta propuesta, enmarcada en la

extensión universitaria, pretende abrir una conversación entre los actores participantes

en el debate pedagógico público

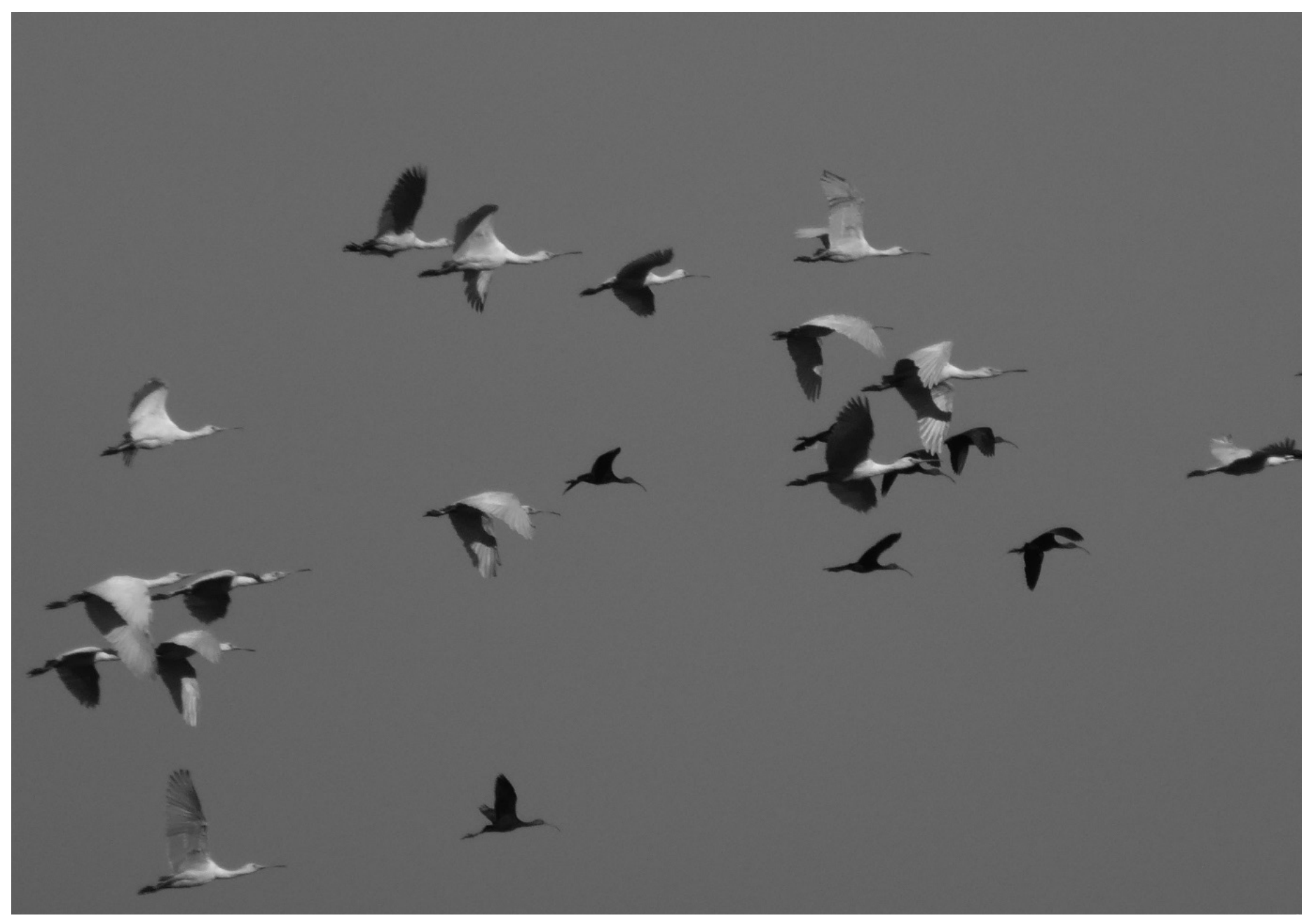

๑) Laura Malachesky 
criterios de validación, en los que el equipo está actualmente profundizando su exploración, se vinculan con la resolución de los problemas abordados, con la posibilidad de generar mayores comprensiones y conocimientos sobre las problemáticas y temas a trabajar, y conciernen también a las relaciones generadas entre los investigadores y todos los participantes involucrados en el proceso (Anderson y Herr, 2007).

Respecto de las dificultades encontradas en el desarrollo de la experiencia de la Red desde el año 2010, están relacionadas con los tiempos y ritmos de cada una de las instituciones involucradas (por ejemplo, el desarrollo de los proyectos UBANEX ${ }^{10}$ se limita a un año de duración). Sin embargo, la propuesta de organizar un espacio participativo, con la modalidad de red, en la que interactúan distintos tipos de organizaciones (municipios, institutos de formación docente, sindicatos, universidades) tensiona la idea de acompasar ritmos y tiempos, y desafía a pensar nuevos modos de articulación y de participación para la toma de decisiones conjuntas.

Asimismo, el proceso de institucionalización de las relaciones de la Red a través de un programa de extensión universitaria planteó tensiones vinculadas a las posiciones de los sujetos en el campo pedagógico, puso sobre la mesa las diversas demandas e intereses de cada uno de los nodos, y a la vez explicitó dificultades y disputas que planteaba el desarrollo de un proceso de organización colectiva frente a las reglas de juego y la cultura institucional universitaria.

\section{La Red desde uno de sus nodos: reconstruyendo un proceso de investigación coparticipada en La Matanza}

Con un camino de experiencias compartidas con la Región Educativa $N^{\circ} 3$ de La Matanza, provincia de Buenos Aires, entre los años 2011 y $2013,{ }^{11}$ comenzamos a desplegar el proyecto denominado "Documentación narrativa de experiencias pedagógicas en redes de investigación-formación-acción entre docentes, directivos e inspectores de la Jefatura Regional $N^{\circ} 3$ del Municipio de La Matanza", que tendría por objetivo reconstruir la memoria pedagógica del sistema escolar del distrito a partir de los relatos de experiencias a través de la conformación de una red de escuelas y colectivos de docentes que investigan sus prácticas pedagógicas. El eje articulador de la propuesta consistió en la producción guiada y sistemática de relatos pedagógicos, la reflexión individual y colectiva en torno a ellos, y su interpretación a través de la construcción colaborativa de tematizaciones. La convocatoria fue llevada adelante por las supervisoras y directoras de todas las modalidades y niveles del sistema escolar de La Matanza, quienes asumieron la posición de coordinadoras del proceso. Durante el transcurso del mismo, los y las docentes participantes se abocaron a la escritura de sucesivas versiones de sus relatos de experiencias pedagógicas. La modalidad que adoptaron dichos encuentros, el lugar y la frecuencia fueron cuestiones acordadas al interior de cada pequeño grupo (conformado por entre cinco y ocho docentes), teniendo en cuenta que el apoyo de la Jefatura Regional habilitó la disposición de tiempos y espacios que, de otro modo, hubieran sido difíciles de establecer.

En cada sesión del dispositivo de documentación narrativa, a partir de la guía de las coordinadoras, se dispusieron rondas de lectura de los relatos en sus diferentes versiones y de comentarios de pares a estas producciones, a fin de ofrecer preguntas, comentarios y sugerencias, para enriquecer las decisiones de escritura y profundizar en la indagación de la experiencia identificada y seleccionada con vistas a avanzar en la reescritura del relato. Fue así que, para mediados del año 2013, eran más de 80 los docentes y directivos de La Matanza que narraban sus experiencias pedagógicas. Y también lo hacían las 22 coordinadoras, quienes habían comenzado a reflexionar acerca del proceso de reposicionamiento que implicaba asumir esa posición en el proyecto de investigación-formación-acción docente. Dichas reflexiones pudieron ser compartidas en el marco de las sesiones de un seminario-taller en el que las supervisoras, junto al equipo de coordinación general, formularon interrogantes con relación a la función del coordinador de procesos de documentación narrativa. En este sentido, se planteó la importancia de su papel para propiciar lecturas y comentarios entre los docentes y, al mismo tiempo, cuidar el modo en que ellas mismas hacían comentarios sobre los comentarios de los colegas para evitar "moldear", desde la "investidura del cargo", las reflexiones de los narradores. Las narrativas de los docentes permitieron capturar las decisiones, los afanes y los saberes que ellos ponen en juego en su hacer pedagógico cotidiano, como también mirar de cerca lo que se enseña y se aprende en los espacios de formación que habitan junto con sus alumnos, con otros docentes, directivos
10) Programa de Subsidios para Proyectos de Extensión Universitaria cuya implementación data del año 2006 en la UBA.

11) Se trata de tres proyectos sucesivos coordinados por integrantes del
Programa de Extensión, implementados en articulación con la Universidad Pedagógica de la Provincia de Buenos Aires (UNIPE), la Dirección Provincial de Nivel Inicial y la Dirección General de Investigación Educativa de la
Provincia de Buenos Aires: "Relatos de experiencia, docentes y prácticas pedagógicas en el nivel inicial” (2011), "Reconstrucción narrativa y autobiográfica de experiencias docentes de La Matanza, San Martín y Pilar" (2012) y "Memoria pedagógica e innovación educativa en el nivel inicial. Documentación narrativa de experiencias de innovación pedagógica en redes de investigación-formación-acción entre docentes" (2013 y 2014). 
y supervisores. Los relatos fueron un medio formidable para que los docentes socialicen los saberes que construyen en sus experiencias pedagógicas, y también una vía para comprender lo que les sucede cuando llevan adelante sus prácticas en determinados contextos institucionales y culturales.

Los relatos de los docentes trazaron un posible mapa de la forma escolar a partir de la descripción de los modos en que viven y transitan por ella. Lo escolar se configura como "territorio" en tanto construcción que en la práctica adopta características singulares. Un territorio en disputa con sentidos heterogéneos, inclusive al interior de cada relato. Asimismo, las narraciones dan cuenta del desafío permanente que asumen los docentes al buscar conformar una escuela abierta como espacio compartido, transitado y apropiado por la comunidad (de y para ella), por los vecinos, por el barrio. El adentro y afuera no encuentran muros definidos y las dinámicas escolares acontecen en tiempos y espacios plurales: se abre lugar a la novedad, se crean otras formas de transmitir la cultura y de alojar a los y las estudiantes y sus realidades complejas, heterogéneas y situadas.

En otro conjunto de relatos aparece el proyecto como "forma de organización" de la intervención pedagógica, como motor de cambio, como potencia. En dichas narraciones, el curriculum se hace presente como instancia relacional, flexible, abierta. Los docentes dan cuenta del "curriculum vivido", nos hablan de ámbitos de creación, recreación y resignificación que configuran una trama, donde lo emergente cobra un lugar fundamental como disparador del proyecto. El curriculum es pensado y transitado como experiencia. Por último, encontramos que las producciones nos relatan los desafíos asumidos ante la irrupción de "nuevas presencias" en el cotidiano escolar. En tanto experiencia, el oficio de enseñar se ve interpelado por el Otro. Los docentes nos invitan a revisar las tensiones intrínsecas a la heterogeneidad de las constituciones grupales. Nos hablan de una escuela que se ensancha, se expande y se dirime entre sus marcas fundacionales y las diversidades que ingresan sin golpear a la puerta.

Es posible advertir que los relatos son parte fundamental del proceso, pero su escritura no fue un acontecimiento aislado de lo que el dispositivo propuso como recorrido que persigue el desarrollo profesional de los docentes. Partimos de la premisa de que esta modalidad de trabajo colectivo, con y entre docentes, permite incorporar nuevas miradas y enfoques acerca de lo que implica ser y constituirse en docente en determinados contextos y bajo ciertas

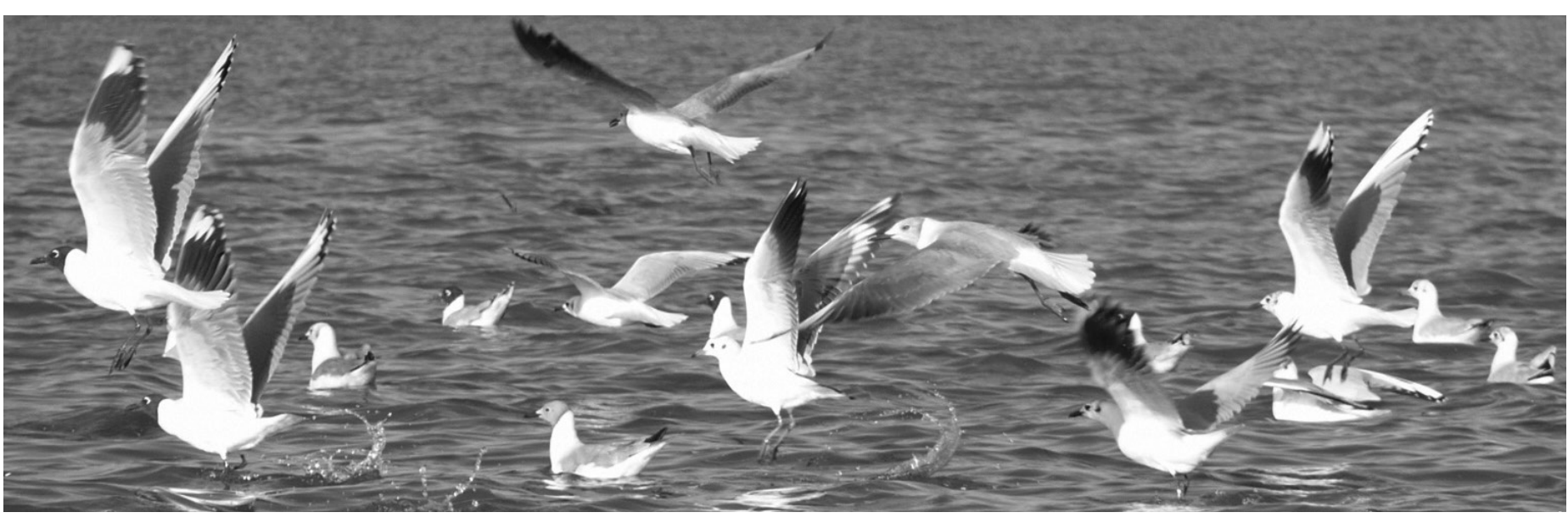

(c) Laura Malachesky 
circunstancias, sin que esto implique necesariamente agregar a sus repertorios de estrategias y formas de pensamiento conceptos abstractos, desprendidos y de la propia experiencia, sino más bien generar y ayudar a resolver nuevas inquietudes, preguntas y recursos en el lenguaje de la práctica, con sus propias palabras y modos de nombrar y pensar la experiencia educativa. A su vez, el dispositivo habilitó para los docentes una posición distinta de la habitual: la de actores centrales de la producción pedagógica y como autores protagonistas en la tarea de contar sus experiencias pedagógicas. Es posible concebir a los relatos como materiales inigualables para conocer lo que hacen, piensan y sienten los que habitan y hacen la escuela. En tanto narraciones profesionales que problematizan el acontecer escolar desde la perspectiva de sus actores, son materiales documentales densamente significativos que llaman e incitan a la reflexión, la conversación informada, la interpretación, el intercambio y la discusión horizontal entre docentes. $Y$ en tanto materiales comunicables que pueden ser acopiados y puestos a circular, manifiestan potencialidades inéditas para la reconstrucción de la memoria pedagógica de la escuela (Suárez, 2011).
Para la Red, esta experiencia significó el desarrollo de un proceso de investigación-formación acción coparticipado que involucró a dos nodos con lógicas político-institucionales heterogéneas: una región educativa entramada con el sistema educativo local y una universidad. Concebir este proyecto implicó tejer vínculos dispuestos a la negociación de las decisiones acerca de aquello que iba aconteciendo en territorio, activó desplazamientos, movimientos y reposicionamientos de los sujetos involucrados en tanto el dispositivo tensionó las habituales relaciones jerárquicas del aparato burocrático escolar y universitario al fomentar lazos horizontales, que lejos de negar las diversas posiciones de poder y saber, las problematiza y reflexiona críticamente sobre ellas (Suárez et al., 2014).

\section{Un cierre que abre nuevas reflexiones: hacia otra pedagogía de la extensión universitaria}

Como consecuencia del trabajo de la Red, cada vez más docentes se han interesado en contar lo que (les) sucede con la enseñanza, en preguntarse y conversar sobre lo que hacen y piensan, en

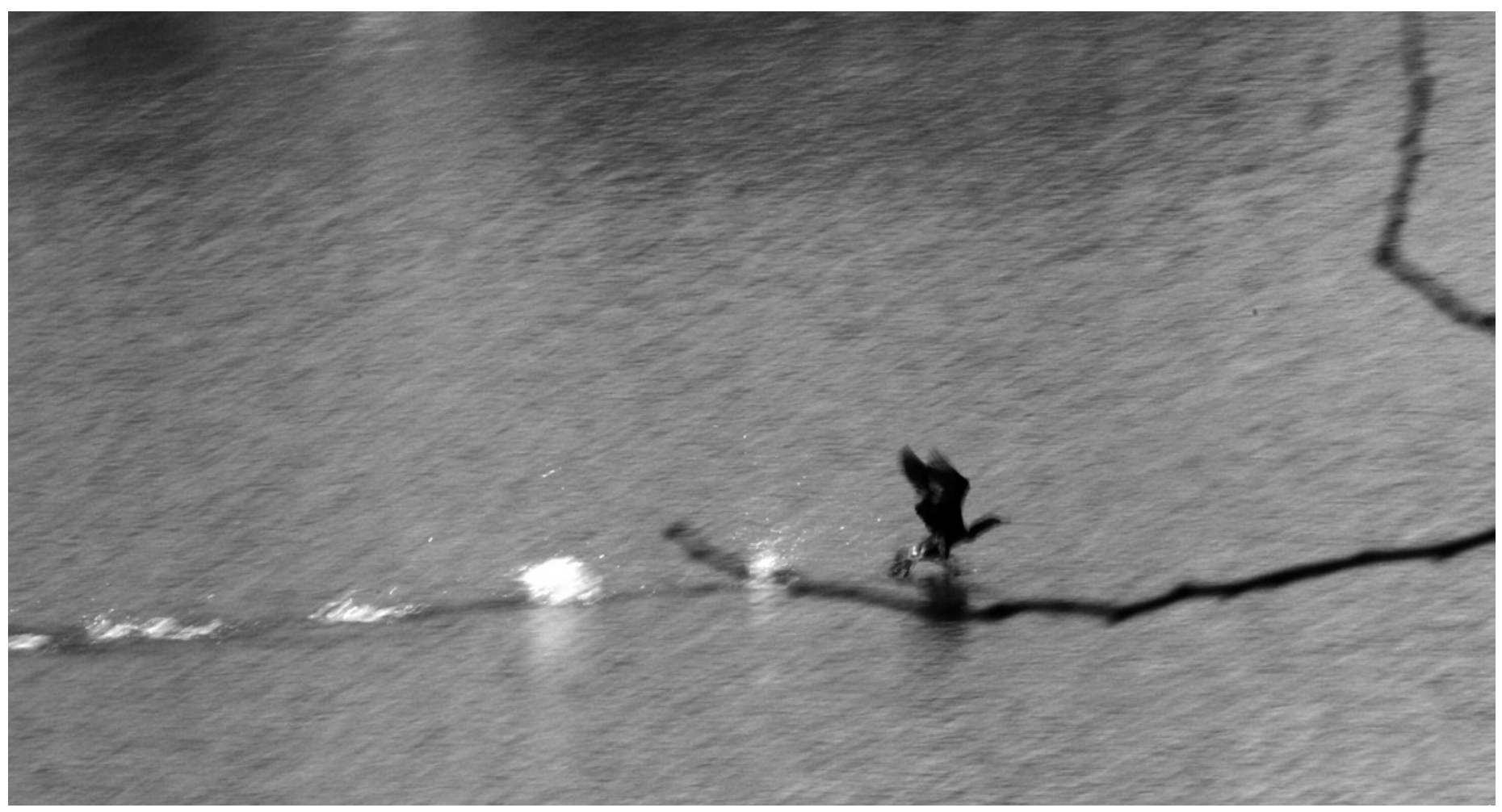

(c) Laura Hormaeche 
reflexionar e indagar sobre sus mundos pedagógicos y lo que interpretan de ellos. $Y$ junto con ese número creciente de docentes narradores e indagadores, el volumen y la densidad del corpus de relatos de experiencias también se fue multiplicando, diversificando, ampliando. $Y$ también los espacios de encuentro, debate y discusiones pedagógicas, donde las voces de los docentes y los relatos de experiencias fueron cobrando centralidad, a través de la participación de diversos actores de una conversación, interpretación y resignificación de las prácticas y saberes mediados por el relato.

Desde esta experiencia podemos sostener que la universidad no solo se ocupa, en algunos casos, de la formación inicial de docentes sino que también, a través de la extensión, puede ocuparse de la formación permanente de maestros y profesores. En este sentido, entenderla de modo "en-redado" nos puede ayudar a pensar de manera ampliada el territorio de la formación docente, más allá de las clasificaciones mediante las que se organiza e institucionaliza la educación, y así trazar otros territorios y visibilizar otros actores colectivos que se desempañan como protagonistas en procesos formativos y de producción de saber pedagógico. Como señala Fernandes (2005), el territorio se constituye, al mismo tiempo, como una convención y una confrontación: dado que posee límites y fronteras, es un espacio de disputa y conflicto. El ámbito de las redes resulta un espacio privilegiado para construcción de saberes en tanto configura una comunidad pedagógica entre los sujetos que la conforman y tensiona y redefine la identidad docente al posicionarse como productora y portadora de saberes pedagógicos. De este modo, los colectivos y redes de docentes pueden resultar un espacio propicio para que tenga lugar la discusión en torno a nuevos criterios para la producción del saber pedagógico, su validación y puesta en circulación desde modalidades colectivas.

A partir del camino recorrido, de los lazos tejidos con los participantes, de las dificultades y los desafíos planteados, destacamos el papel preponderante de las prácticas extensionistas en la visibilización de experiencias, sujetos y saberes en el campo pedagógico. Y sostenemos la necesidad de ampliar y profundizar el debate en torno de la extensión universitaria como ámbito de enseñanza, formación, intervención y producción de conocimientos críticos desde los territorios. Se trata, en definitiva, de examinar las posibilidades para el desarrollo de otra pedagogía de la extensión universitaria que la piense como una posibilidad para habilitar la conversación con sujetos y experiencias que se inscriben en y hacen el territorio; esto es, en un campo de posiciones y disposiciones de saber y de poder dinámicas y relacionales.

\section{Referencias bibliográficas}

Anderson, G.; Herr, K. (2007). El docente investigador: la investigación-acción como una forma válida de generación de conocimientos. En Sverdlick, I. (Comp.), La investigación educativa. Una herramienta de conocimiento y acción. Buenos Aires: Novedades Educativas.

Dávila, P.; Argnani, A. (2015). Redes pedagógicas y colectivos docentes conformados en torno de la documentación narrativa de experiencias pedagógicas. Linhas, Dossiê "Narrativas, (auto)biografias e educação", 16(32), 72-92. doi: 10.5965/1984723816322015072.

Dávila, P.; Argnani, A. (2017). Tejiendo lazos y articulaciones: formación, investigación e intervención desde la extensión universitaria. En Memorias de las $1^{\circ}$ Jornadas sobre las prácticas docentes en la universidad pública. Transformaciones actuales y desafíos para los procesos de formación. Secretaría de Asuntos Académicos, Universidad Nacional de La Plata.

Fernandes, B. M. (2005). Movimentos socioterritoriais e movimentos socioespaciais. Contribuçao teórica para uma leitura geográfica dos movimentos sociais. Revista OSAL, (16, enero- abril), 273-283. Buenos Aires: CLACSO.

Martínez Boom, A.; Peña Rodríguez, F. (2009). Instancias y Estancias de la Pedagogía. La Pedagogía en movimiento. Bogotá: Universidad de San Buenaventura. Souza, E. C. de (2011). Acompañamiento, mediación biográfica y formación de formadores: dimensiones de investigación-formación. Educación y Pedagogía, 23(61, septiembre-diciembre), 41-56.

Suárez, D. H. (2009). Relatos pedagógicos, docentes e investigación narrativa de la experiencia escolar: aportes de la investigación cualitativa y colaborativa para la formación y el desarrollo profesional de los docentes. Tesis Doctoral no publicada. Universidad de Buenos Aires.

(2011). Relatos de experiencia, saber pedagógico y reconstrucción de la memoria escolar. En Alliaud, A.; Suárez, D. H. (Coords.), El saber de la experiencia: narrativa, investigación y formación docente. Buenos Aires: CLACSO y Facultad de Filosofía y Letras, Universidad de Buenos Aires.

— (2014). Espacio (auto)biográfico, investigación educativa y formación docente en Argentina: un mapa imperfecto de un territorio en expansión. Revista Mexicana de Investigación Educativa, 19(62), 763-786. México DF.

Suárez, D. H.; Argnani, A. (2011). Nuevas formas de organización colectiva y producción de saber pedagógico: la Red de Formación Docente y Narrativas Pedagógicas. Revista da FAEEBA: educação e contemporaneidade, 20(36), 43-56. Salvador.

Suárez D. H.; Dávila, P.; Argnani, A. (2015). Trabajo docente y extensión universitaria: la experiencia de la red de formación docente y narrativas pedagógicas. En Actas del III Seminario Nacional de la Red ESTRADO Argentina: "Formación y trabajo docente: aportes a la democratización educativa". Facultad de Humanidades y Ciencias de la Educación, Universidad Nacional de La Plata.

Suárez, D.; Vassiliades, A.; Dávila, P.; Argnani, A.; Caressa, Y. (2014). Una experiencia colectiva de formación, investigación e intervención en el campo educativo: la Red de Formación Docente y Narrativas Pedagógicas". En Actas del VI Congreso Nacional de Extensión Universitaria. Universidad Nacional de Rosario. Universidad Pedagógica Nacional (2002). Memorias. III Encuentro Iberoamericano de Colectivos Escolares y Redes de Maestros que hacen Investigación desde su Escuela. Santa Marta, Colombia. 